EPJ Web of Conferences 70, 00034 (2014)

DOI: $10.1051 /$ epjconf/ 20147000034

(C) Owned by the authors, published by EDP Sciences, 2014

\title{
New bottomonium(-like) resonances' spectroscopy and decays at Belle
}

\author{
Umberto Tamponi ${ }^{1,2, a}$ \\ ${ }^{1}$ Universitá degli Studi di Torino \\ ${ }^{2}$ INFN, Sezione di Torino
}

\begin{abstract}
We report the recent progresses in the study of bottomonium spectroscopy and decays obtained with the $\Upsilon(1 S), \Upsilon(2 S)$, and $\Upsilon(5 S)$ samples collected by the Belle experiment. Among these results, particular interest is generated by the observation, with high significance, of the missing singlet states $h_{b}(1 P)$ and $h_{b}(2 P)$ in $\Upsilon(5 S)$ hadronic decays. The transition $\Upsilon(5 S) \rightarrow \pi^{+} \pi^{-} h_{b}(1,2 P)$ in particular is found to be mediated by two new, bottomonium-like, charged state $Z_{b}$ and $Z_{b}^{\prime}$, whose nature is still argument of discussion. The large samples of $h_{b}(1 P)$ and $h_{b}(2 P)$ provided by these transitions allow the word most precise measurement of the hyperfine splitting in the $1 S$ system and the first measurement of this quantity in the $2 S$ system, together with a new high significance observation of the $\eta_{b}(1 S)$ and a new, precise measurements of its parameters. The study of the radiative transition $h_{b}(2 P) \rightarrow \eta_{b}(2 S)$ resulted in the first evidennce of the $2 S$ singlet state. We report also new studies of the $\Upsilon(n S) \rightarrow \eta \Upsilon(m S)$ transitions, the results of an intensive search for bottomonium decays to charmonium and the first observation of $\Upsilon(1 S)$ and $\Upsilon(2 S)$ decaying in low multiplicity, mesonic final states.
\end{abstract}

\section{Observation of $b \bar{b}$ singlet states in $\Upsilon(5 S) \rightarrow \pi^{+} \pi^{-} X$}

\subsection{The $\Upsilon(5 S)$ as a source of single states}

The Belle experiment [1] at the KEKB $e^{+} e^{-}$asymmetric collider [2] collected the world largest samples of $\Upsilon(1 S), \Upsilon(2 S)$ and $\Upsilon(5 S)$. The $\Upsilon(5 S)$ sample revealed a totally unexpected potential for the study of the lower bottomonium resonances, in particular the singlet states $h_{b}$ and $\eta_{b}$. The starting point of this study is an inclusive analysis of the of the missing mass $M_{\text {miss }}\left(\pi^{+} \pi^{-}\right)$from the $\pi^{+} \pi^{-}$ pair in $\Upsilon(5 S) \rightarrow \pi^{+} \pi^{-}+X$ final states [3]. Once the large combinatorial background is fitted and subtracted, the remaining distribution (Figure 1) shows different peaks, due to different dipion transitions: the $h_{b}(1 P)$ and $h_{b}(2 P)$ signals are clearly visible with a significance of $6.2 \sigma$ and $12.4 \sigma$ respectively, together with the $\Upsilon(5 S) \rightarrow \pi^{+} \pi^{-} \Upsilon(n S)$ signals.

The measured masses of the $h_{b}$ states are $M_{h_{b}(1 P)}=9898.3 \pm 1.1_{-1.1}^{+1.0} \mathrm{MeV} / \mathrm{c}^{2}$ and $M_{h_{b}(2 P)}=$ $10259.8 \pm 0.6_{-1.0}^{+0.4} \mathrm{MeV} / \mathrm{c}^{2}$. The observation of $\Upsilon(5 S) \rightarrow \Upsilon(1,2,3 S) \pi^{+} \pi^{-}$with such as large branching ratios is itself surprising, since the $b \bar{b}$ states with mass above the threshold for the decay into a pair of

ae-mail: tamponi@to.infn.it

This is an Open Access article distributed under the terms of the Creative Commons Attribution License 2.0, which permits unrestricted use, distribution, and reproduction in any medium, provided the original work is properly cited. 


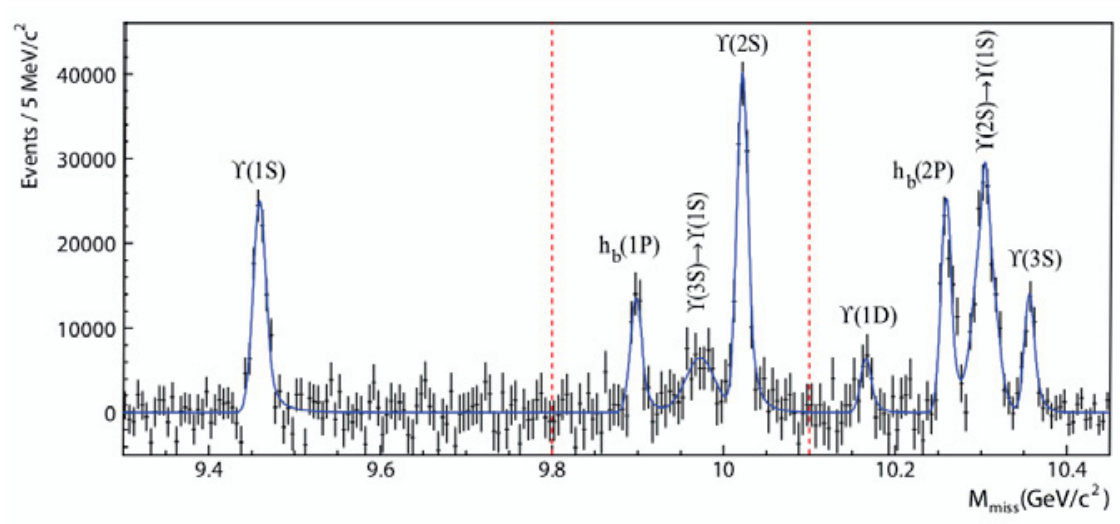

Figure 1. Missing mass in $\Upsilon(5 S) \rightarrow \pi^{+} \pi^{-}+X$ events. The two peaks located at $9.95 \mathrm{GeV} / \mathrm{c}^{2}$ and $10.3 \mathrm{GeV} / \mathrm{c}^{2}$ are due to the known $\Upsilon(2,3 S) \rightarrow \pi^{+} \pi^{-} \Upsilon(1 S)$ transitions.

$B$ mesons are commonly expected not to have large transitions to the lower states. In addition to that, the calculation of $\mathcal{B}\left[\Upsilon(5 S) \rightarrow \pi^{+} \pi^{-} h_{b}(1 P)\right]$ includes amplitudes related to a spin flip of the $b$ quark, whose presence is predicted by non-relativistic QCD (NRQCD) to suppress this transition with respect to a non spin-flipping process such as $\Upsilon(5 S) \rightarrow \pi^{+} \pi^{-} \Upsilon(n S)$. The measured ratio $\frac{\mathcal{B}\left(\Upsilon(5 S) \rightarrow \pi^{+} \pi^{-} h_{b}(1 P)\right)}{\mathcal{B}\left(\Upsilon(5 S) \rightarrow \pi^{+} \pi^{-} Y(1 S)\right)}=$ 0.77 , surprisingly indicates no significant suppression. This unexpected behavior can be explained with the introduction of an intermediate charged state that mediates the dipion transition, with the decay chain $\Upsilon(5 S) \rightarrow \pi^{ \pm} Z_{b}^{\mp} \rightarrow \pi^{+} \pi^{-} h_{b}(1,2 P)$; the resulting two-body transitions $\Upsilon(5 S) \rightarrow \pi^{ \pm} Z_{b}^{\mp}$ and $Z_{b}^{\mp} \rightarrow \pi^{\mp} h_{b}(1,2 P)$ are now not spin-flipping. Experimentally this hypothetical $Z_{b}$ state can be detected reconstructing only the two pions and exploiting the missing mass distributions: it should be seen as a peak in the $h_{b}$ yield distribution, calculated using the missing mass from the second pion, when computed in bins of the missing mass from the first pion. A double peak observed in
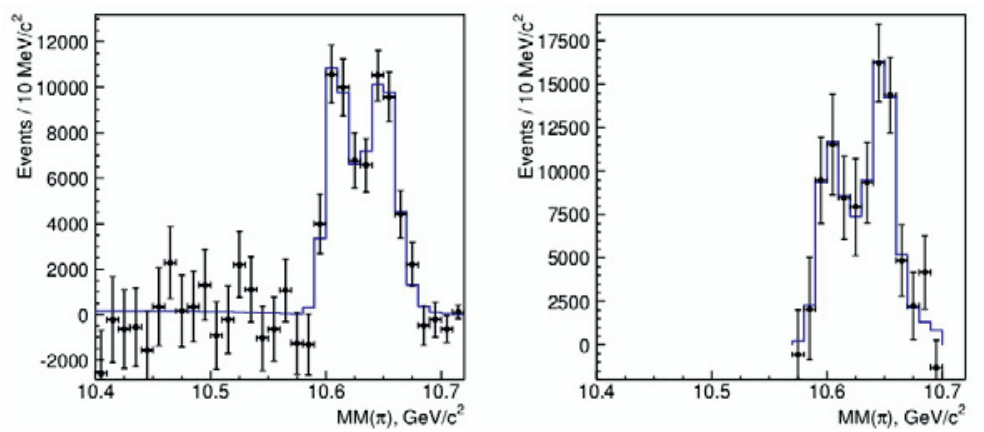

Figure 2. $h_{b}(1 P)$ (left) and $h_{b}(2 P)$ (right) yield in bins of recoil mass against the single charged pion, in $\Upsilon(5 S) \rightarrow$ $\pi^{+} \pi^{-}+X$ events..

both the distributions of the $h_{b}(1 P)$ and $h_{b}(2 P)$ (Figure 2) yields, is thus interpreted as a doublet of charged states $Z_{b}$ and $Z_{b}^{\prime}$, with masses $M_{Z_{b}}=10607.2 \pm 2.0 \mathrm{MeV} / \mathrm{c}^{2}, M_{Z_{b}^{\prime}}=10652.2 \pm 1.5 \mathrm{MeV} / \mathrm{c}^{2}$ and widths $\Gamma_{Z_{b}}=18.4 \pm 2.4 \mathrm{MeV}, \Gamma_{Z_{b}^{\prime}}=11.5 \pm 2.2 \mathrm{MeV}$ [4]. The same structures are also observed 
in the exclusive final states $\pi^{+} \pi^{-} \Upsilon(1,2,3 S)$ where the $\Upsilon$ states are reconstructed in the leptonic final state $\Upsilon(n S) \rightarrow \mu^{+} \mu^{-}$. The $Z_{b}$ and $Z_{b}^{\prime}$ parameters extracted in this five independent final states are in agreement, providing a strong observation of these states.

\subsection{Study of $\eta_{b}(1 S)$ and $\eta_{b}(2 S)$}

The ground state of bottomonium, $\eta_{b}(1 S)$ is predicted to be largely produced in $h_{b}$ decays, but it was observed both by CLEO and BaBar studying the radiative transition $\Upsilon(2,3 S) \rightarrow \gamma \eta_{b}(1 S)$. This kind of studies are affected by different sources of irreducible background, mainly the $\Upsilon(1 S)$ production via Initial State Radiation (ISR), the $\Upsilon(2 S) \rightarrow \gamma \chi_{b}(1 P)$ and the $\Upsilon(2,3 S) \rightarrow \pi^{0} \pi^{0} \Upsilon(1 S)$ transitions. The unexpected high number of $h_{b}$ and the strong experimental signature provided by the charged pions due to the presence of the $Z_{b}$ intermediate state allow, for the first time, the study of the radiative decay $h_{b}(1 P) \rightarrow \gamma \eta_{b}(1 S)$, opening new perspectives on the study of the $\eta_{b}(1 S)$ itself [5].

The $h_{b}(1 P) \rightarrow \gamma \eta_{b}(1 P)$ transition is studied requiring a single pion missing mass compatible with $Z_{b}$ mass and fitting the $h_{b}$ yield, extracted from the missing mass from the $\pi^{+} \pi^{-}$ system $M_{\text {miss }}\left(\pi^{+} \pi^{-}\right)$, in bins of $\Delta M_{\text {miss }}$, where $\Delta M_{\text {miss }}=$ $M_{\text {miss }}\left(\pi^{+} \pi^{-} \gamma\right)-M_{\text {miss }}\left(\pi^{+} \pi^{-}\right)+M\left(h_{b}\right), M_{\text {miss }}\left(\pi^{+} \pi^{-} \gamma\right)$ is the missing mass from the $\pi^{+} \pi^{-} \gamma$ system, and $M\left(h_{b}\right)$ is the measured $h_{b}$ mass. $\Delta M_{\text {miss }}$ peaks, by construction, at the $\eta_{b}$ mass value.

With this technique an observation of the $\eta_{b}$ is possible without reconstructing its decays. The significance of the $\eta_{b}(1 S)$ peak is $15 \sigma$ in the $h_{b}(2 P) \rightarrow \gamma \eta_{b}(1 S)$ and $9 \sigma$ in $h_{b}(1 P) \rightarrow \gamma \eta_{b}(1 S)$ (Fig. 3), and such a clean observation allows to measure with unprecedented precision the parameters of this resonance, obtaining $M_{\eta_{b}(1 S)}=9401.0 \pm 1.9_{-2.4}^{+1.4}$ $\mathrm{MeV} / \mathrm{c}^{2}$ and $\Gamma_{\eta_{b}(1 S)}=12.4_{-5.6-3.4}^{+5.5+11.5} \mathrm{MeV}$. The theoretical importance of such measurements is connected to the hyperfine splitting in the $1 S$ system, defined as the mass difference between the $\Upsilon(1 S)$ and the $\eta_{b}(1 S)$. Previous measurement fixed this value slightly above the theoretical prediction obtained from both NRQCR and potential based models. The new value $\Delta M_{H F}(1 S)=\left(59.3 \pm 1.9_{-1.2}^{+1.6}\right) \mathrm{MeV} / \mathrm{c}^{2}$ obtained by the Belle collaboration is lower than the previous ones and in agreement with the non-relativistic QCD predictions. The same technique can be applied also in the reconstruction of the $h_{b}(2 S)$ (Fig. 3). In this case the significance of $4.9 \sigma$, allows to archive the first evidence of this resonance and the first measurements of its parameters. The hyperfine splitting in the $2 S$ system is thus measured for the first time, obtaining the value of $\Delta M_{H F}(2 S)=\left(23.4_{-4.5}^{+4.0}\right) \mathrm{MeV} / \mathrm{c}^{2}$ which is in agreement with the theoretical predicitons.
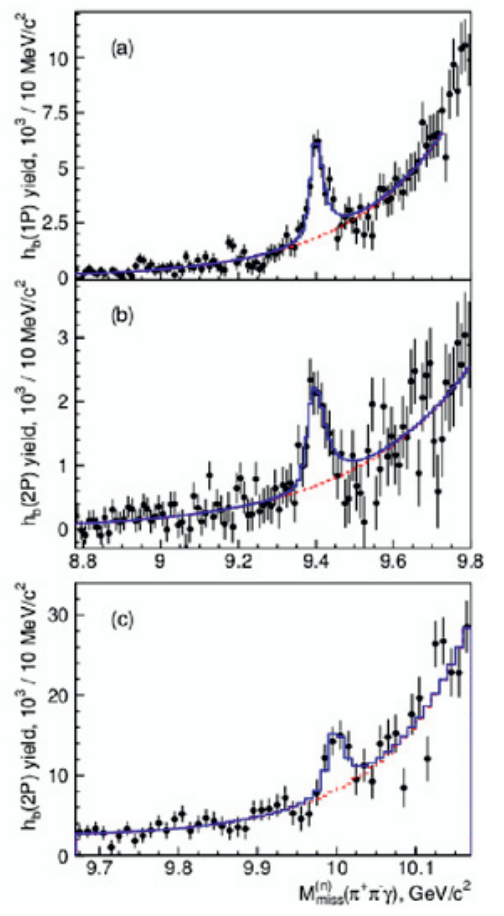

Figure 3. $h_{b}(2 P)$ and $h_{b}(2 P)$ yields in bins of $\Delta M_{\text {miss }}$, in the $\eta_{b}(1 S)$ (top) and $\eta_{b}(2 S)$ (bottom) regions. 


\section{Progresses in $\Upsilon(n S) \rightarrow \eta \Upsilon(m S)$}

In the QCD Multipole Expansion (QCDME) formalism [6] the $\eta$ transitions between $\Upsilon$ states is modeled as a two gluon process, with either two M1, or one E1 and one M2 gluon: both cases imply a spin flip of the $b$ quark. The corresponding amplitude should scale as $1 / m_{b}$, leading to a suppression of these processes. By scaling from the $\psi^{\prime} \rightarrow \eta J / \psi$ transition, one expects $\mathbf{B}[\Upsilon(2 S) \rightarrow \eta \Upsilon(1 S)]=8 \times 10^{-4} . \Upsilon(2,3,4 S) \rightarrow \eta \Upsilon(1 S)$ branching ratios have been measured by BaBar [7, 8] and CLEO [9]: they are either unexpectedly large $(\Upsilon(4 S))$, or too small $(\Upsilon(2 S)$ and $\Upsilon(3 S))$. We search for the $\Upsilon(2 S) \rightarrow \eta \Upsilon(1 S)$ process with the exclusive reconstruction of the $\eta$ in both the $\gamma \gamma$ and the $\pi^{+} \pi^{-} \pi^{0}$ mode. The $\Upsilon(1 S)$ is reconstructed in the leptonic modes $e^{+} e^{-}$and $\mu^{+} \mu^{-}$. The measured branching ratio is $\mathcal{B}[\Upsilon(2 S) \rightarrow \eta \Upsilon(1 S)]=(3.41 \pm 0.28 \pm 0.35) \times 10^{-4}$, with a high significance $\eta$ signal observed (Fig. 4).

This value is in good agreement with the latest theoretical predictions [10][11], even if a $20 \%$ discrepancy still persists. The isospin violating transition $\Upsilon(2 S) \rightarrow \pi^{0} \Upsilon(1 S)$ is investigated too, with the same technique, but no signal is observed and and upper limit is set: $\mathcal{B}\left(\Upsilon(2 S) \rightarrow \pi^{0} \Upsilon(1 S)\right)<4.6$ (90\% CL).

The $\eta$ transition is observed for the first time from $\Upsilon(5 S)$ too, requiring also in this case the exclusive reconstruction of $\Upsilon(1,2 S) \rightarrow \mu^{+} \mu^{-}$(Fig. 4). We measure the branching ratios $\mathcal{B}[\Upsilon(5 S) \rightarrow \eta \Upsilon(1 S)]=$ $(7.3 \pm 1.6($ stat $) \pm 0.8($ syst $)) \times 10^{-4}$ and $\mathcal{B}[\Upsilon(5 S) \rightarrow \eta \Upsilon(2 S)]=(38 \pm 4($ stat $) \pm 5($ syst $)) \times 10^{-4}$, surprisingly higher than the branching fraction for the $2 S \rightarrow 1 S$ transition.
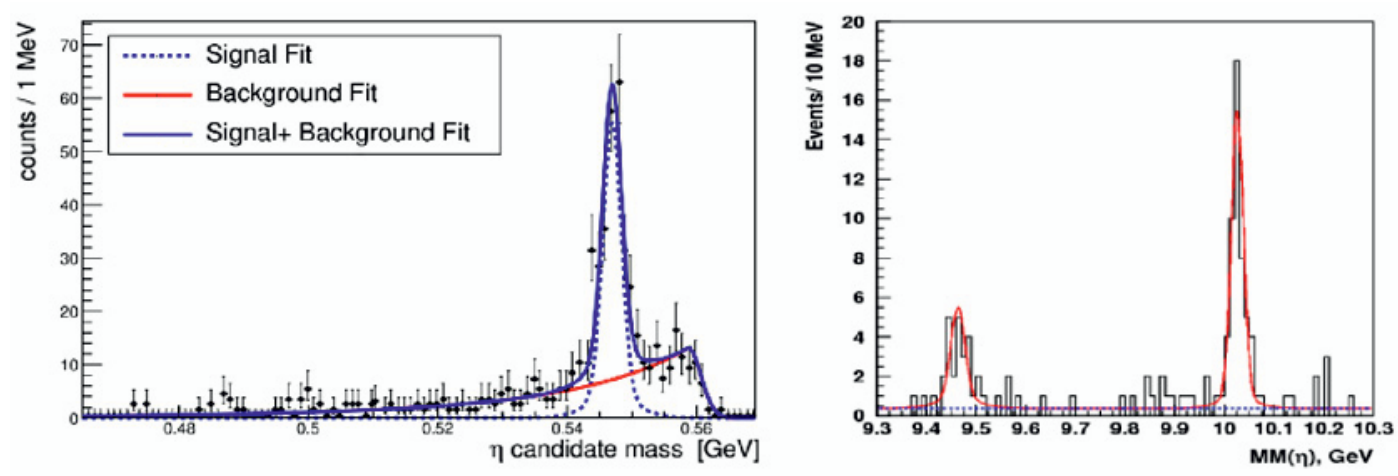

Figure 4. $\eta$ invariant mass in $\Upsilon(2 S) \rightarrow \eta \Upsilon(1 S)$ events (left) and recoil mass against $\eta \rightarrow \pi^{+} \pi^{-} \pi^{0}$ candidates in $\Upsilon(5 S)$ decays (right) 


\section{$3 \Upsilon(1,2 S)$ and $\chi_{b}(1 P)$ decays}

A large number of studies of the charmonium production in bottomonium decays were carried on by Belle during last year.

In particular intensive studies of the radiative transitions $\Upsilon(1,2 S) \rightarrow$ $\gamma c \bar{c}$ and of the double charmonium production $b \bar{b} \rightarrow(c \bar{c})(c \bar{c})$ led to a large set of upper limits. In details a systematic search for $\eta_{c}, \chi_{c J}$, $X(3872), X(3915), X(4350)$, and $Y(4140)$ signals in $\Upsilon(2 S)$ radiative decays is performed by Belle [12], extending a previous work on the $\Upsilon(1 S)$ transitions to charmonium states [13]. A search for new resonances is performed too, looking for the $\gamma_{R} \gamma \psi^{\prime}$ final state where $\gamma_{R}$ is the radiative photon from the $\Upsilon(2 S)$ decay. No signal is observed and upper limits are set for all the transitions at the $10^{-4}$ level.

The second field of research is the double charmonium production, i.e. the $b \bar{b} \rightarrow(c \bar{c})(c \bar{c})$ decays [14]. Recent studies report theoretical prediction on $b \bar{b} \rightarrow$ $J / \psi J / \psi, J / \psi \psi^{\prime}, \psi^{\prime} \psi^{\prime}$, where $b \bar{b}=$ $\Upsilon(1 S), \Upsilon(2 S), \chi_{b J}(1 P)$, obtained with different frameworks: light cone formalism (LCF), potential
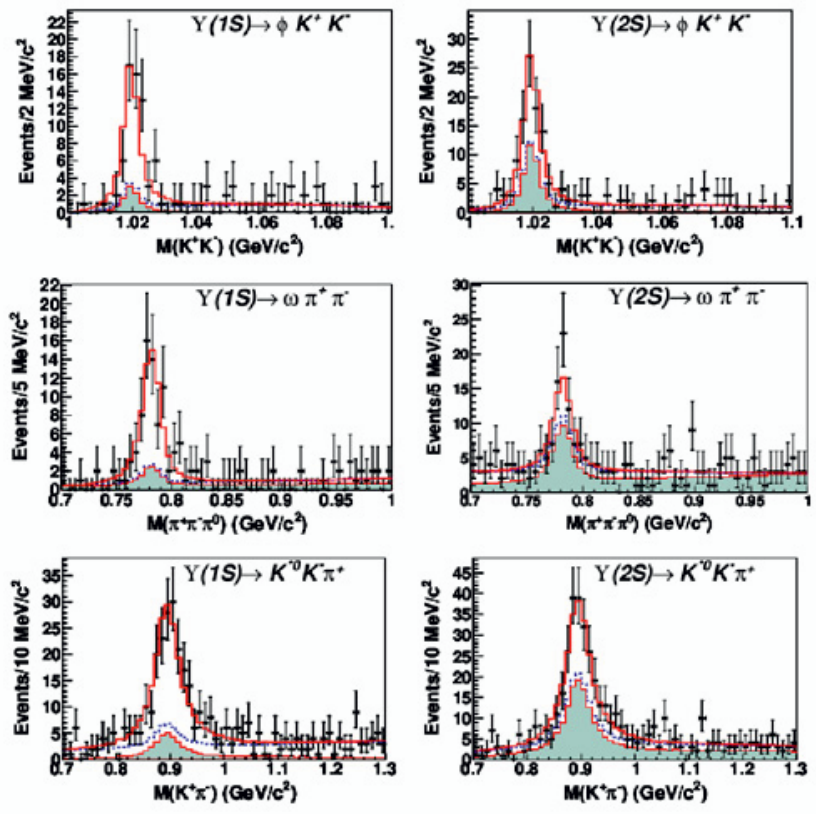

Figure 5. The $K^{+} K^{-}, \pi^{+} \pi^{-} \pi^{0}$, and $k^{+} \pi^{-}$invariant-mass distributions for the candidate events from $\Upsilon(1 S)$ and $\Upsilon(2 S)$ three-body decays. Solid points are data, open histograms show the best fits, dashed curves are the total background estimates, and shaded histograms are the normalized continuum background contributions.

QCD (pQCD), and non-relativistic QCD (NRQCD). The $J / \psi$ candi-

dates are reconstructed in the $\mu^{+} \mu^{-}$and $e^{+} e^{-}$final states, while the $\psi^{\prime}$ is reconstructed in the dominant decay $\pi^{+} \pi^{-} J / \psi \cdot \chi_{b 0}(1 P), \chi_{b 1}(1 P)$, and $\chi_{b 2}(1 P)$ are distinguished by the energy of the photon from the radiative transition $\Upsilon(2 S) \rightarrow \gamma \chi_{b J}(1 P)$. Signals are observed neither in the full reconstruction of the bottomonium decays, nor in the missing mass from the $J / \psi$ candidate distribution, and stringent upper limits are set. This upper limits are in agreement with the NRQCD predictions, while they are significantly below the light cone formalism and pQCD ones. The last field of investigation is the search of $\Upsilon(1 S)$ and $\Upsilon(2 S)$ decays with few bodies, mesonic final states [15]. In the charmonium sector, according to the so called $12 \%$ rule, given a final state $\mathrm{X}$ the ratio between the branching fractions $Q_{c}=\frac{\psi(2 S) \rightarrow X}{J / \psi \rightarrow X}$ is predicted to be 0.12 . This rule, that can be derive from the NRQCD, is know to be violated in some Vector-Tensor and Vector-Pseudoscalar final state, leading to the so called " $\rho$ - $\pi$ puzzle". A similar rule can be derived also in the bottomonium, obtaining $Q_{b}=\frac{\Upsilon(2 S) \rightarrow X}{\Upsilon(1 S) \rightarrow X}=0.77$, and this value is expected to be even more precise than $Q_{c}$, due to the non relativistic nature of bottomonium. In order to check for the first time this predictions, the Belle collaboration studied ten low multiplicity channels: $\phi f_{2}^{\prime}, \omega f_{2}, \rho a_{2}, K^{* 0} \bar{K}_{2}{ }^{* 0}, K_{1}(1270)^{+} K^{-}, K_{1}(1400)^{+} K^{-}, b_{1}(1235)^{+} \pi^{-}, \phi K^{+} K^{-}$, 
$\omega \pi^{+} \pi^{-}, K^{* 0} K^{-} \pi^{+}$. The two body-channels are chosen on order to have the Vector-Tensor or AxialPseudoscalar combination. In five of these final states $\left(K^{* 0} \bar{K}_{2}{ }^{* 0}, K_{1}(1400)^{+} K^{-}, \phi K^{+} K^{-}, \omega \pi^{+} \pi^{-}\right.$, $\left.K^{* 0} K^{-} \pi^{+}\right)$signals are observed with a significance greater than $6 \sigma$, in some cases in both $\Upsilon(1 S)$ and $\Upsilon(2 S)$ (Fig. 5). The resulting ratios $Q_{b}$ are in agreement with the expected value except for the $\omega \pi^{+} \pi^{-}$, in which the upper limit of $Q_{b}<0.55$ is $2.5 \sigma$ below the theoretical prediction.

\section{Conclusions}

The last year was an exciting year for bottomonium physics. In January, 2011, only one singlet state was known, $\eta_{b}(1 S)$, thanks to the studies performed by CLEO and BaBar, and the only way to measure its parameters seemed to be the study of the rare radiative transitions from the $\Upsilon$ states below the open beauty threshold. The discovery of the charged pion transition from the $\Upsilon(5 S)$ has given a sudden boost the the studies in this sector, with the observation of $h_{b}(1,2 S)$ and $\eta_{b}(1 S)$ and the first evidence of $\eta_{b}(2 S)$ with a very clean experimental signature. The contextual observation of the bottomoniumlike states $Z_{b}$ and $Z_{b}^{\prime}$, which are thought to be $B B$ and $B B^{*}$ molecular states, is opening perspectives of a completely new spectroscopy. From a theoretical point of view the NRQCD is confirming itself as one of the best model available, giving good predictions of both decay branching fraction (first measurement of the $Q$ ratio in the bottomonium sector), transitions amplitudes ( $\Upsilon(2 S) \rightarrow \eta \Upsilon(1 S)$ ), and hyperfine splittings of the $1 S$ and $2 S$ states. The full comprehension of the $\eta$ transition still remains an open problem, in particular the transitions from the $\Upsilon$ states above the open beauty threshold.

\section{References}

[1] A. Abashian et al., Nucl. Instrum. Meth. A479, 117-232 (2002).

[2] S. Kurokawa, E. Kikutani, Nucl. Instrum. Meth. A 499,1 (2003) and other papers included in this volume.

[3] I. Adachi et al. [Belle Collaboration], Phys. Rev. Lett. 108 (2012) 032001

[4] A. Bondar et al. [Belle Collaboration], Phys. Rev. Lett. 108 (2012) 122001

[5] Belle. Collaboration, arXiv:1205.6351v1 [hep-ex].

[6] T. -M. Yan, Phys. Rev. D22, 1652 (1980).

[7] B. Aubert et al. [ The BaBar Collaboration ], Phys. Rev. D78, 112002 (2008).

[8] J. P. Lees, et al. [ The BaBar Collaboration ], arXiv:1108.5874v1 [hep-ex].

[9] Q. He et al. [ CLEO Collaboration ], Phys. Rev. Lett. 101, 192001 (2008).

[10] M. B. Voloshin, Prog. Part. Nucl. Phys. 61, 455 (2008).

[11] Y. -P. Kuang, Front. Phys. China 1, 19 (2006).

[12] X. L. Wang et al. [ Belle Collaboration ], arXiv:1108.4514v1 [hep-ex].

[13] C. P. Shen et al. [ Belle Collaboration ], Phys. Rev. D82, 051504(R) (2010).

[14] C. P. Shen, C. Z. Yuan, T. Iijima and B. Collaboration, Phys. Rev. D 85 (2012) 071102

[15] C. P. Shen, C. Z. Yuan, T. Iijima and B. Collaboration, arXiv:1205.1246v1 [hep-ex] 